\title{
Association between the expression levels of TAZ, AXL and CTGF and clinicopathological parameters in patients with colon cancer
}

\author{
SHU-DONG ZHANG ${ }^{1,2^{*}}$, CIAN M. MCCRUDDEN ${ }^{2 *}$, HIU-FUNG YUEN ${ }^{3}$, \\ KA LAI LEUNG ${ }^{1,2}$, WAN-JIN HONG ${ }^{3}$ and HANG FAI KWOK ${ }^{1,2,4}$ \\ ${ }^{1}$ Faculty of Health Sciences, University of Macau, Macau, SAR, P.R. China; \\ ${ }^{2}$ Center for Cancer Research and Cell Biology, School of Pharmacy, Queen's University Belfast, Belfast BT9 7BL, UK; \\ ${ }^{3}$ Institute of Molecular and Cell Biology, Agency for Science, Technology and Research, Proteos, Singapore 138673, \\ Republic of Singapore; ${ }^{4}$ Department of Oncology, University of Cambridge, Cambridge CB2 0XZ, UK
}

Received December 18, 2014; Accepted October 9, 2015

DOI: 10.3892/ol.2015.3999

\begin{abstract}
Colon cancer accounts for a large proportion of all the cancer-associated morbidities worldwide. Genetic analysis and stratification of patients based on survival may identify genetic signatures potentially useful for prognostic or treatment planning purposes. Previous studies have reported that the messenger (m)RNA expression levels of tafazzin (TAZ), AXL receptor tyrosine kinase (AXL) and connective tissue growth factor (CTGF) were able to predict the survival of patients with colon cancer in two independent colon cancer datasets. However, limited clinicopathological data were available from these two datasets. By contrast, a large colon cancer dataset comprising 566 patients has been recently published in the Gene Expression Omnibus database, which contains data regarding tumor stage and location, and genetic status of mismatch repair (MMR), Kirsten rat sarcoma viral oncogene homolog (KRAS), B-Raf proto-oncogene serine/threonine kinase (BRAF) and tumor protein p53 (TP53). In the present study, the mRNA expression levels of TAZ, AXL and CTGF were evaluated, and the TAZ-AXL-CTGF signature was correlated with the available pathological parameters and survival data. Overexpression of TAZ, AXL and CTGF was observed to be associated with severe pathological stage, deficiency in MMR, colon cancer subtype $\mathrm{C} 4$ and mutations in the BRAF gene. In addition, overexpression of TAZ-AXL-CTGF was associated with short overall survival in patients with mutations in the TP53 gene, colon cancer subtype C6, proficient MMR and wild-type status of the KRAS and BRAF genes. Furthermore, the prognostic value of TAZ-AXL-CTGF overexpression
\end{abstract}

Correspondence to: Professor Hang Fai Kwok, Faculty of Health Sciences, University of Macau, Avenida de Universidade, Macau, SAR, P.R. China

E-mail:hfkwok@umac.mo

${ }^{*}$ Contributed equally

Key words: colon cancer, TAZ, AXL, CTGF, prognosis, survival was observed to be independent of all the clinicopathological parameters and mutational statuses analyzed. The results of the present study confirm the previously reported findings, and suggest that the TAZ-AXL-CTGF mRNA signature is a potential prognostic indicator in colon cancer.

\section{Introduction}

The morbidity rates exhibited by patients with colon cancer are among the highest of all types of cancer. Therefore, the identification of novel prognostic markers for colon cancer is important for appropriate treatment planning, in order to ensure that patients receive more efficacious and less toxic treatment regimens (1). Recent advances in colon cancer research have led to the identification of Kirsten rat sarcoma viral oncogene homolog (KRAS) and neuroblastoma RAS viral oncogene homolog as negative predictive markers, and to the introduction of novel treatment agents, including bevacizumab and anti-epidermal growth factor receptor (EGFR)s, which have resulted in median overall survival $>30$ months in patients with metastatic colorectal cancer (2). Despite these advances, no positive predictors for response and treatment outcome in these patients have been identified thus far. For early colon cancer, microsatellite instability is a well-known prognostic factor. However, the identification of further prognostic markers has experienced limited success in recent years (3). Tafazzin (TAZ), also known as WW domain containing transcription regulator 1 (WWTR1), is an important transcription coactivator and a key downstream effector of the mammalian Hippo signaling pathway (4), which participates in the development of colon cancer $(5,6)$.

The use of the messenger (m)RNA expression levels of TAZ and its downstream factors AXL receptor tyrosine kinase (AXL) and connective tissue growth factor (CTGF) as a three-gene signature for the prognosis of patients with colon cancer has been previously reported in two independent cohort datasets (5). However, these datasets did not contain clinicopathological data of the patients, contrarily to the cohort Gene Expression Omnibus Series (GSE)40967, which has been recently submitted to the Gene Expression Omnibus (GEO) database (http://www.ncbi.nlm.nih. 
Table I. Clinicopathological features of the patients in the dataset GSE40967.

\begin{tabular}{lcc}
\hline Characteristics & No. of cases & $\%$ \\
\hline Age, years & 566 & $68(22-97)$ \\
$\quad$ Median (range) & & \\
Gender & & 45.2 \\
Female & 256 & 54.8 \\
Male & 310 & \\
Tumor stage & & 0.71 \\
0 & 4 & 5.83 \\
1 & 33 & 46.64 \\
2 & 264 & 36.22 \\
3 & 205 & 10.60 \\
4 & 60 &
\end{tabular}

Tumor location

$\begin{array}{lll}\text { Proximal } & 224 & 39.6 \\ \text { Distal } & 342 & 60.4\end{array}$

Colon cancer subtype

\begin{tabular}{lrr} 
C1 & 116 & 20.495 \\
C2 & 104 & 18.375 \\
C3 & 75 & 13.251 \\
C4 & 59 & 10.424 \\
C5 & 152 & 26.855 \\
C6 & 60 & 10.600 \\
MMR status & & \\
Proficient & 444 & 78.4 \\
Deficient & 75 & 13.3 \\
Missing & 47 & 8.3 \\
BRAF status & & \\
Wild-type & 461 & 81.4 \\
Mutant & 51 & 9.0 \\
Missing & 54 & 9.5 \\
KRAS status & & 38.0 \\
Wild-type & 328 & 38.3 \\
Mutant & 217 & 38.7 \\
Missing & 21 & 28.4 \\
TP53 status & & 33.6 \\
Wild-type & 161 & \\
Mutant & 190 & \\
Missing & 215 & \\
\hline
\end{tabular}

GSE, Gene Expression Omnibus Series; C1, chromosome instability-immune down; C2, deficient MMR subtype; C3, KRAS mutant subtype; C4, cancer stem cell subtype; C5, chromosome instability-int/Wingless-up subtype; C6, chromosome instability-normal-like subtype; MMR, mismatch repair; BRAF, B-Raf proto-oncogene serine/threonine kinase; KRAS, Kirsten rat sarcoma viral oncogene homolog.

gov/geo/) (7). At present, no other GEO database regarding colon cancer cohorts contains more patients for whom gene expression and patient relapse-free survival outcome data are available than the aforementioned GSE40967 cohort, which
A

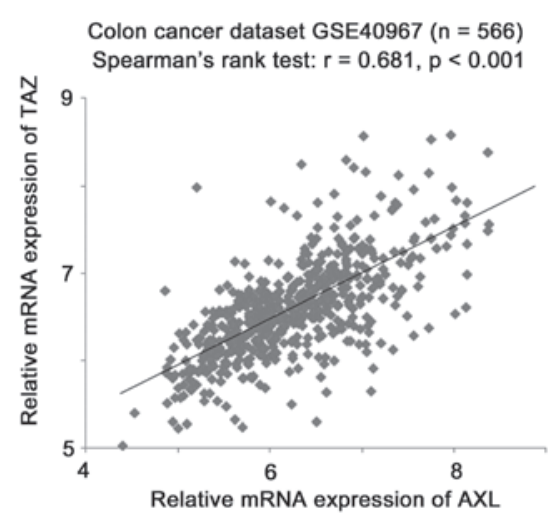

B

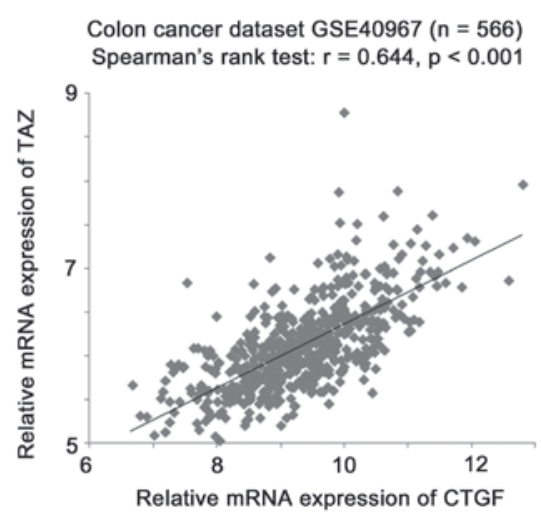

C

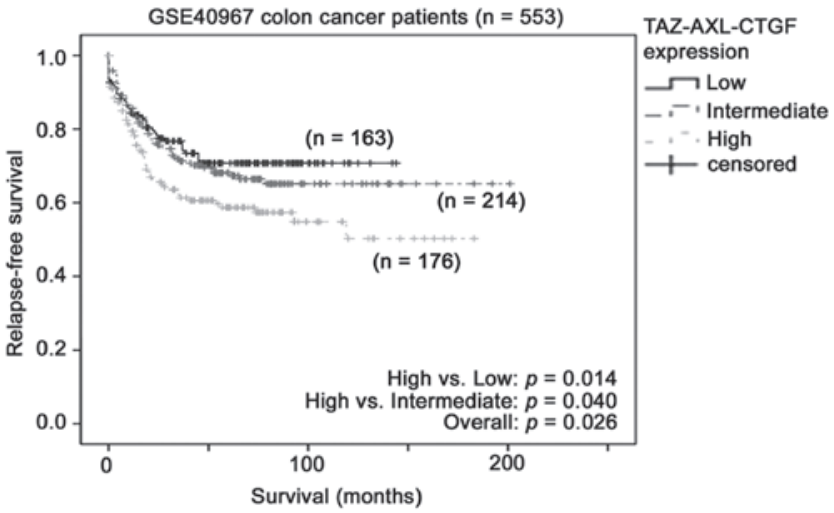

Figure 1. Correlation between survival and the expression levels of TAZ, AXL and CTGF in the dataset GSE40967. Correlation between the mRNA expression levels of TAZ and the mRNA expression levels of (A) AXL and (B) CTGF in patients with colon cancer. (C) Association between the TAZ-AXL-CTGF expression signature and relapse-free survival in patients with colon cancer. TAZ, tafazzin; CTGF, connective tissue growth factor; mRNA, messenger RNA; GSE, Gene Expression Omnibus Series.

describes a number of clinicopathological parameters of 566 patients with colon cancer, including age, gender, tumor stage and location (distal or proximal), survival outcome and response to adjuvant chemotherapy. This dataset also details genetic parameters of the patients, including status of mismatch repair (MMR) and mutational status of KRAS, B-Raf proto-oncogene serine/threonine kinase (BRAF) and tumor protein p53 (TP53) genes. Due to this combination of genetic and clinicopathological data, GSE40967 may be a powerful tool for confirming the prognostic potential of the TAZ-AXL-CTGF mRNA expression signature in colon 
A

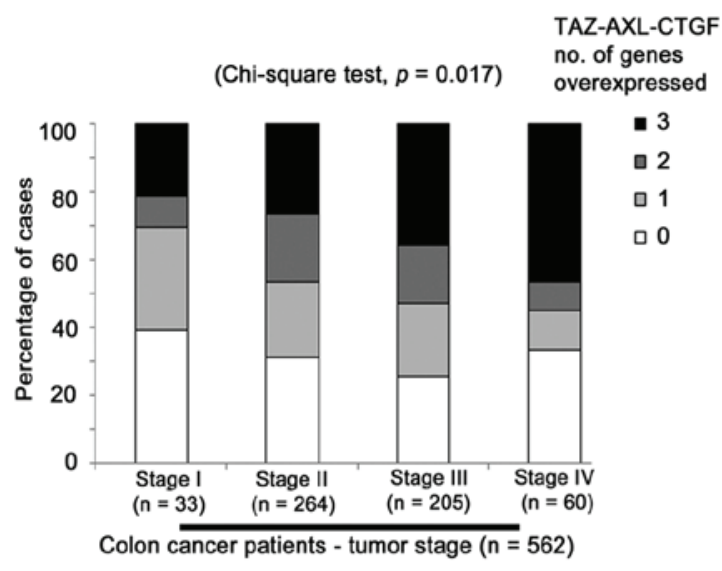

C

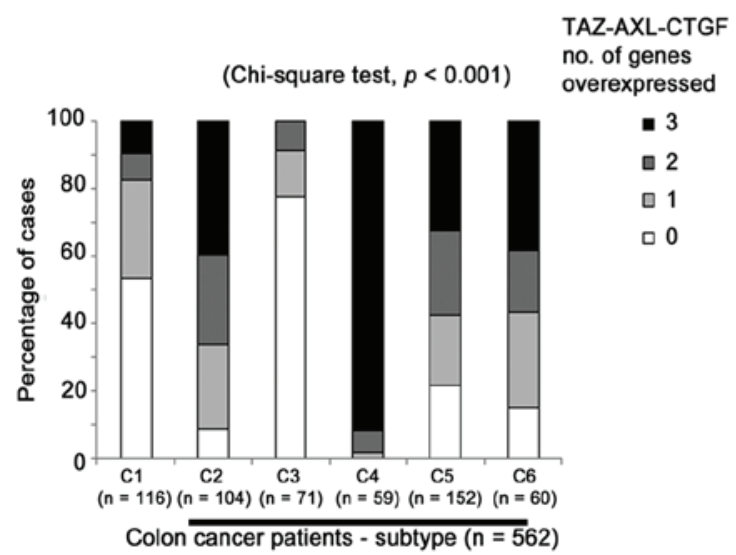

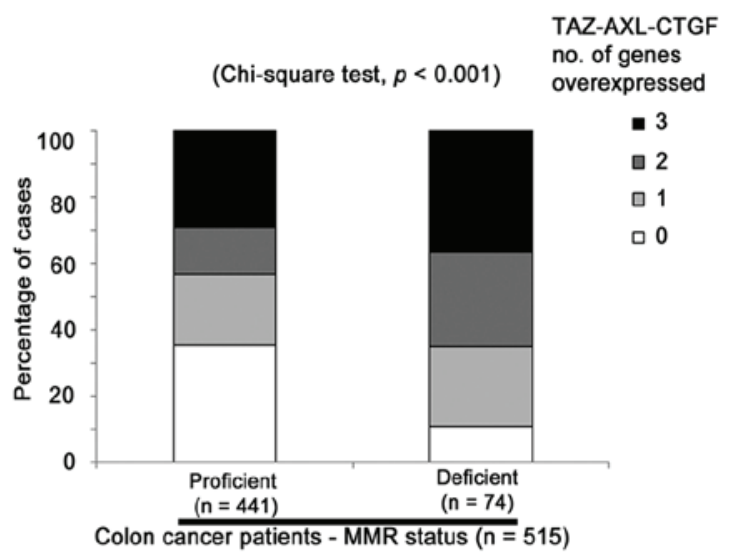

D

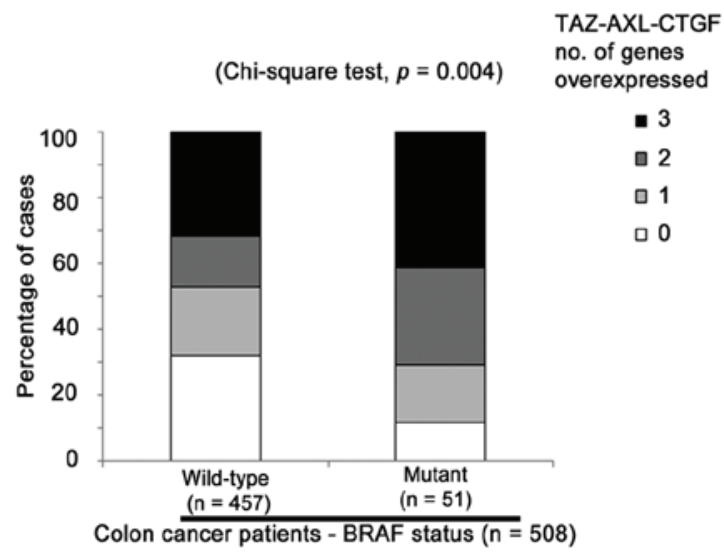

Figure 2. Association between the TAZ-AXL-CTGF expression signature and clinicopathological parameters in patients with colon cancer. Histogram displaying the different TAZ-AXL-CTGF expression signatures observed in patients stratified based on their (A) tumor stage, (B) MMR status, (C) tumor subtype classification and (D) BRAF mutational status. TAZ, tafazzin; CTGF, connective tissue growth factor; MMR, mismatch repair; BRAF, B-Raf proto-oncogene, serine/threonine kinase.

cancer. In addition, the comprehensive information provided by GSE40967 may enable to determine the association between the aforementioned genetic and clinicopathological characteristics and the prognostic significance of the TAZ-AXL-CTGF signature in patients with colon cancer, which was not possible to test in the previous study by Yuen et al (5).

\section{Materials and methods}

Microarray gene expression data were retrieved from the data matrixes deposited in the GEO database by Marisa et al (7). $\mathrm{R}$ scripting was used to extract the expression values from the probe sets for TAZ, AXL and CTGF, and the clinical data from the data matrixes available at GEO, as previously described (5). All statistical analyses were performed using SPSS software, version 19.0 (IBM SPSS, Armonk, NY, USA). The association between the expression levels of TAZ, AXL and CTGF were analyzed by Spearman's rank test. The mRNA expression levels of these genes were classified as high or low, using the value of the median expression level as the cut-off point. Patients were divided into three groups, based on the expression levels of TAZ, AXL and CTGF, as follows: i) The TAZ-AXL-CTGF-low group consisted of patients who exhibited low expression levels (below the median value) of these three genes; ii) the TAZ-AXL-CTGF-high group consisted of patients who displayed high expression levels (above the median value) of these three genes; and iii) the TAZ-AXL-CTGF-intermediate group consisted of patients who presented other expression patterns of the above three genes. The survival time of patients stratified by this grouping method were analyzed by Kaplan-Meier and Cox regression analyses. Multivariate Cox regression analysis was performed including all the available clinicopathological parameters, genetic statuses and mRNA expression levels of TAZ, AXL and CTGF, using a forward conditional stepwise regression method with an entry criteria of $\mathrm{P}<0.05$, which was considered to indicate a statistically significant difference. The clinicopathological data of the patients are detailed in Table I. The sample size was different for each analysis due to variations in the data available for each patient.

\section{Results and Discussion}

As represented in Fig. 1, a significantly positive correlation was observed between the mRNA expression levels of TAZ and the mRNA expression levels of AXL (Spearman's rank test, $\mathrm{r}=0.681, \mathrm{P}<0.001, \mathrm{n}=566$; Fig. $1 \mathrm{~A}$ ) and CTGF (Spearman's rank test, $\mathrm{r}=0.644, \mathrm{P}<0.001, \mathrm{n}=566$; Fig. 1B). These findings 
A

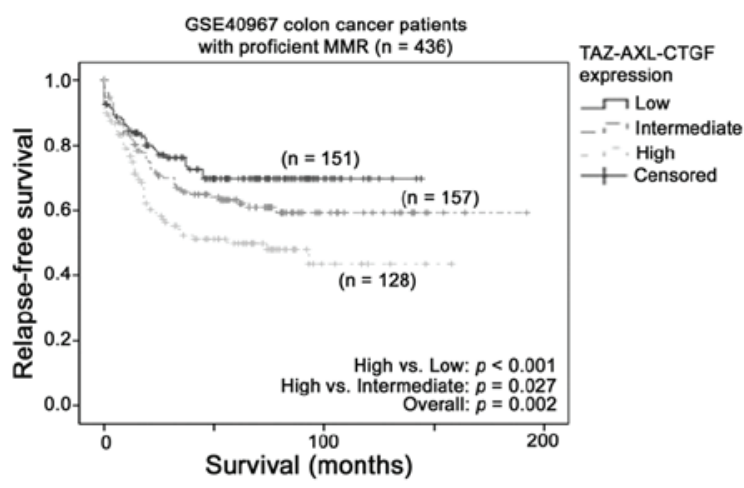

C

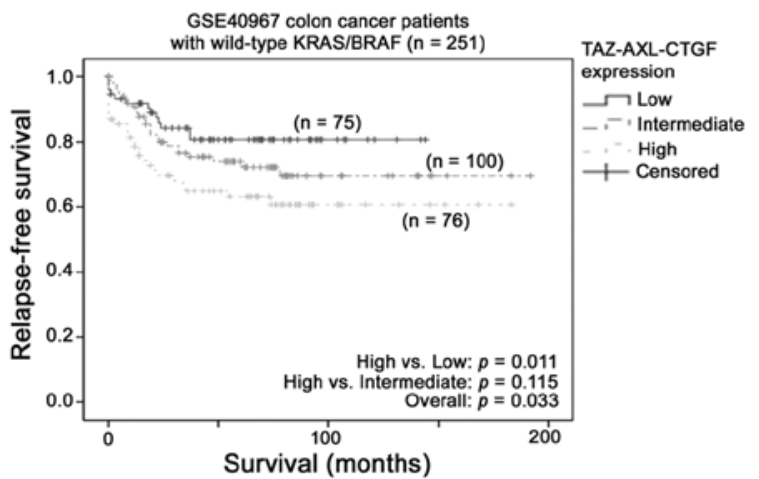

$\mathbf{E}$

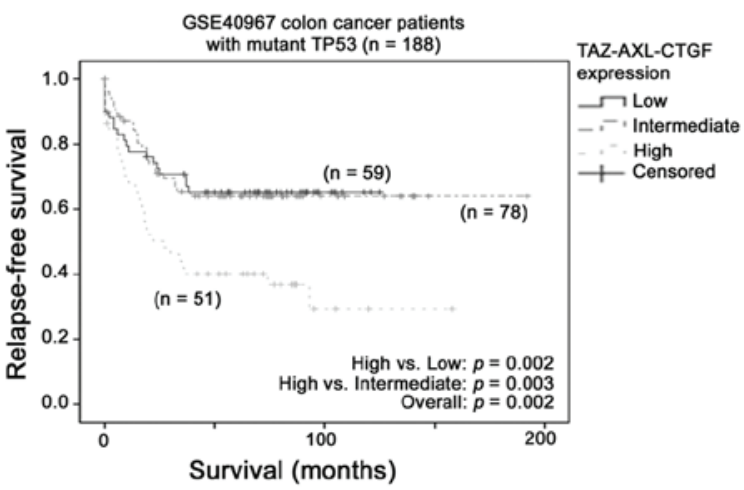

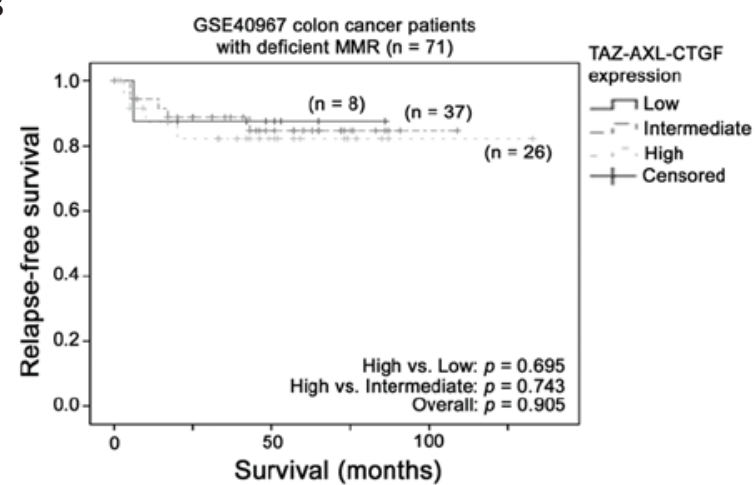

D

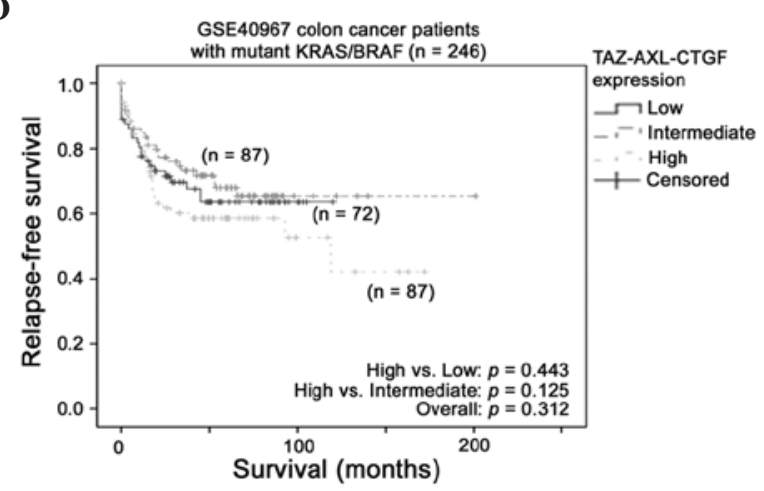

$\mathbf{F}$

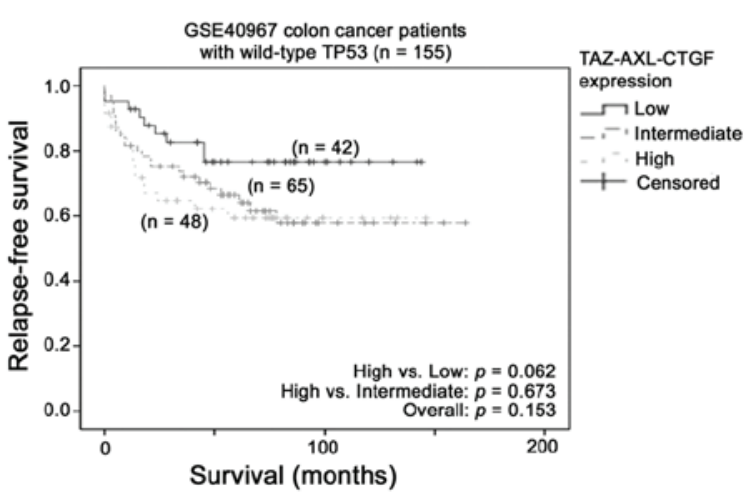

Figure 3. Kaplan-Meier survival curves representing the association between the expression levels of TAZ-AXL-CTGF and survival in patients with (A) proficient MMR, (B) deficient MMR, (C) wild-type KRAS/BRAF, (D) mutant KRAS/BRAF, (E) wild-type TP53 and (F) mutant TP53. TAZ, tafazzin; CTGF, connective tissue growth factor; MMR, mismatch repair; KRAS, Kirsten rat sarcoma viral oncogene homolog; BRAF, B-Raf proto-oncogene serine/threonine kinase; TP53, tumor protein $\mathrm{p} 53$.

A

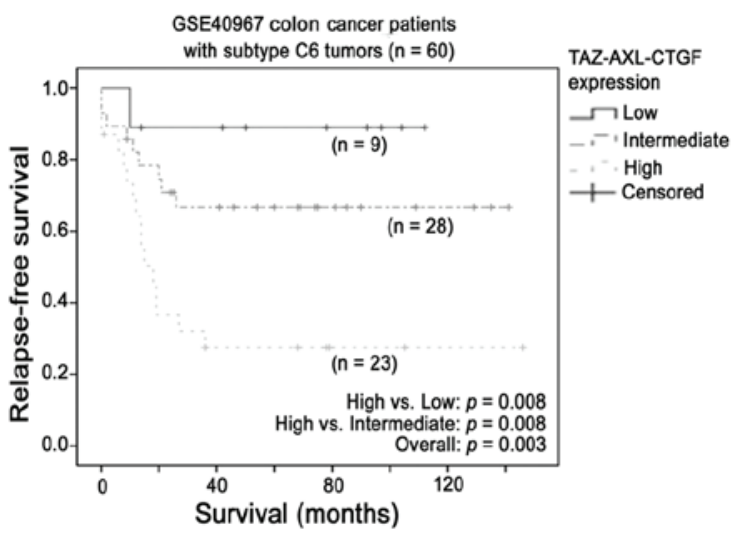

B

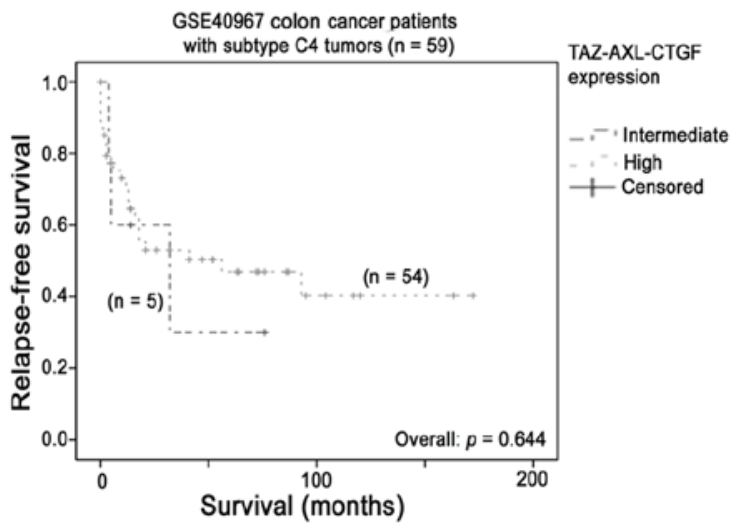

Figure 4. Kaplan-Meier survival curves representing the association between the expression levels of TAZ-AXL-CTGF and survival in patients with subtype (A) C6 and (B) C4 tumors. TAZ, tafazzin; CTGF, connective tissue growth factor. 
Table II. Cox regression analysis of survival of patients with colon cancer.

\begin{tabular}{|c|c|c|c|c|}
\hline \multirow{2}{*}{$\begin{array}{l}\text { Clinicopathological } \\
\text { variables }\end{array}$} & \multicolumn{2}{|c|}{ Univariate analysis } & \multicolumn{2}{|c|}{ Multivariate analysis } \\
\hline & Hazard ratio $(95 \% \mathrm{CI})$ & P-value & Hazard ratio $(95 \% \mathrm{CI})$ & P-value \\
\hline Age $(n=552)$ & $1.000(0.989-1.011)$ & 0.945 & & \\
\hline \multicolumn{5}{|l|}{ Gender } \\
\hline Female $(\mathrm{n}=249)$ & 1 & Reference & & \\
\hline Male $(n=304)$ & $1.271(0.941-1.717)$ & 0.115 & & \\
\hline \multicolumn{5}{|l|}{ Tumor stage } \\
\hline $\mathrm{I}(\mathrm{n}=32)$ & 1 & Reference & 1 & Reference \\
\hline II (n=260) & $7.717(1.069-55.701)$ & 0.043 & $5.232(0.717-38.209)$ & 0.103 \\
\hline III (n=201) & $14.809(2.061-106.430)$ & 0.007 & $6.649(0.911-48.541)$ & 0.062 \\
\hline IV $(n=60)$ & $52.845(7.232-386.123)$ & $<0.001$ & $29.934(3.894-230.075)$ & 0.001 \\
\hline \multicolumn{5}{|l|}{ Adjuvant chemotherapy } \\
\hline No $(n=305)$ & 1 & Reference & & \\
\hline Yes $(n=232)$ & $1.791(1.313-2.443)$ & $<0.001$ & & \\
\hline \multicolumn{5}{|l|}{ Tumor location } \\
\hline Proximal $(n=216)$ & 1 & Reference & & \\
\hline Distal $(n=337)$ & $1.285(0.941-1.754)$ & 0.115 & & \\
\hline \multicolumn{5}{|l|}{ MMR status } \\
\hline Proficient $(n=436)$ & 1 & Reference & 1 & Reference \\
\hline Deficient $(\mathrm{n}=71)$ & $0.361(0.190-0.684)$ & 0.002 & $0.404(0.176-0.930)$ & 0.033 \\
\hline \multicolumn{5}{|l|}{ KRAS status } \\
\hline Wild-type (n=322) & 1 & Reference & & \\
\hline Mutant $(\mathrm{n}=210)$ & $1.436(1.061-1.943)$ & 0.019 & & \\
\hline \multicolumn{5}{|l|}{ BRAF status } \\
\hline Wild-type $(\mathrm{n}=450)$ & 1 & Reference & & \\
\hline Mutant $(\mathrm{n}=49)$ & $0.928(0.525-1.639)$ & 0.504 & & \\
\hline \multicolumn{5}{|l|}{ TP53 status } \\
\hline Wild-type $(\mathrm{n}=155)$ & 1 & Reference & & \\
\hline Mutant $(\mathrm{n}=188)$ & $1.353(0.952-1.925)$ & 0.136 & & \\
\hline \multicolumn{5}{|l|}{ TAZ-AXL-CTGF status } \\
\hline Low $(n=163)$ & 1 & Reference & 1 & Reference \\
\hline Intermediate $(\mathrm{n}=214)$ & $1.138(0.775-1.670)$ & 0.511 & $1.609(0.927-2.792)$ & 0.091 \\
\hline $\operatorname{High}(n=176)$ & $1.609(1.098-2.357)$ & 0.015 & $2.646(1.513-4.627)$ & 0.001 \\
\hline \multicolumn{5}{|l|}{ Colon cancer subtype } \\
\hline $\mathrm{C} 1(\mathrm{n}=114)$ & 1 & Reference & & \\
\hline $\mathrm{C} 2(\mathrm{n}=101)$ & $0.524(0.302-0.912)$ & 0.022 & & \\
\hline $\mathrm{C} 3(\mathrm{n}=67)$ & $0.844(0.485-1.467)$ & 0.547 & & \\
\hline $\mathrm{C} 4(\mathrm{n}=59)$ & $1.920(1.180-3.124)$ & 0.009 & & \\
\hline C5 $(n=152)$ & $0.922(0.599-1.418)$ & 0.712 & & \\
\hline $\mathrm{C} 6(\mathrm{n}=60)$ & $1.394(0.844-2.302)$ & 0.195 & & \\
\hline
\end{tabular}

Survival data was available for 553 patients. The patient numbers do not equal 553 in each univariate analysis due to variation in the availability of data. Missing data was not included in the Cox-regression analysis. CI, confidence interval; MMR, mismatch repair; KRAS, Kirsten rat sarcoma viral oncogene homolog; BRAF, B-Raf proto-oncogene, serine/threonine kinase; TP53, tumor protein p53; TAZ, tafazzin; CTGF, connective tissue growth factor; C1, chromosome instability-immune down; C2, dMMR subtype; C3, KRAS mutant subtype; C4, cancer stem cell subtype; C5, chromosome instability-int/Wingless-up subtype; C6, chromosome instability-normal-like subtype.

are consistent with the fact that TAZ is a transcriptional regulator for AXL and CTGF (5). In addition, high expression levels of TAZ-AXL-CTGF $(n=176)$ were associated with shorter relapse-free survival, compared with intermediate and low expression levels of these three genes (log-rank test, $\mathrm{P}=0.040$ and $0.014, \mathrm{n}=214$ and 163, respectively; Fig. 1C). 
These results are in agreement with the results previously obtained using two colon cancer datasets (5). Similarly to the previous report by Yuen et al (5), the expression levels of TAZ-AXL-CTGF were positively correlated with tumor stage ( $\chi^{2}$ test, $\mathrm{P}=0.017, \mathrm{n}=562$; Fig. $2 \mathrm{~A}$ ). High expression levels of TAZ-AXL-CTGF were also associated with deficiency in MMR ( $\chi^{2}$ test, $\mathrm{P}<0.001, \mathrm{n}=515$; Fig. $2 \mathrm{~B}$ ), colon cancer subtype $\mathrm{C} 4$ ( $\chi^{2}$ test, $\mathrm{P}<0.001, \mathrm{n}=562$; Fig. $\left.2 \mathrm{C}\right)$, and mutant BRAF status ( $\chi^{2}$ test, $\mathrm{P}=0.004, \mathrm{n}=508$; Fig. 2D). The TAZ-AXL-CTGF-high and -low groups were further compared, and differential expression of the MMR-signature-associated genes was identified (8). Thus, the expression levels of genes that are usually overexpressed in MMR-proficient colon cancer, including reduced nicotinamide adenine dinucleotide phosphate oxidase 1, quinolinate phosphoribosyltransferase, 3-hydroxy-3-methylglutaryl-CoA synthase 2, arylsulfatase E, $\gamma$-glutamyl hydrolase, glutathione peroxidase 2, serine peptidase inhibitor Kazal type 1 , transcription factor 7, inhibitor of DNA binding 1, B-cell chronic lymphocytic leukemia/lymphoma 11A, indian hedgehog, guanylate cyclase $2 \mathrm{C}$, protein kinase adenosine monophosphate-activated $\beta 1$, Achaete-Scute complex homolog 2, pleiomorphic adenoma gene-like 2, transcription factor-like 5, kinesin family member 3B, villin 1, cadherin, EGF laminin A seven-pass G-type receptor 3, gap junction protein $\beta 1$, solute carrier family 5 (sodium/glucose cotransporter) member 1 , dipeptidase 1 , transmembrane 9 superfamily protein member 4 and family with sequence similarity 3 member A, were reduced in the TAZ-AXL-CTGF-high group, compared with the TAZ-AXL-CTGF-low group. By contrast, genes that are normally overexpressed in MMR-deficient colon cancer, including dual specificity phosphatase 4 , cysteine-rich protein 1, major histocompatibility complex class I polypeptide-related sequence B, granzyme A, matrix metalloproteinase-12, secreted phosphoprotein 1, v-set and immunoglobulin domain containing 4, uridine phosphorylase 1 and tribbles pseudokinase 2, were significantly overexpressed in the TAZ-AXL-CTGF-high group, compared with the TAZ-AXL-CTGF-low group. These results indicate that the aforementioned genes may be involved in the association between the TAZ-AXL-CTGF signature and the MMR status displayed by patients with colon cancer (data not shown).

A significant association was observed between high expression levels of TAZ-AXL-CTGF and poor relapse-free survival in patients with MMR-proficient colon cancer (log-rank test, $\mathrm{P}=0.002$; Fig. $3 \mathrm{~A})$, but not in those with deficient MMR status (log-rank test, $\mathrm{P}=0.905$; Fig. 3B). Similarly, this TAZ-AXL-CTGF signature was associated with poor relapse-free survival in patients with wild-type KRAS/BRAF (log-rank test, $\mathrm{P}=0.033$; Fig. $3 \mathrm{C}$ ) but not in those with mutant KRAS/BRAF (log-rank test, $\mathrm{P}=0.312$; Fig. 3D). Furthermore, the expression levels of TAZ-AXL-CTGF were associated with poor relapse-free survival in patients with mutant TP53 (log-rank test, $\mathrm{P}=0.002$; Fig. 3E), contrarily to those with wild-type TP53 (log-rank test, $\mathrm{P}=0.153$; Fig. 3F).

A previous study classified colon cancer into six different subtypes, of which, C4 and C6 subtypes were observed to be associated with poor prognosis (7). In the present study, the expression levels of TAZ, AXL and CTGF were significantly associated with poor prognosis in patients with colon cancer subtype C6 (log-rank test, $\mathrm{P}=0.003$; Fig. 4A), but no significant association was observed for subtype $\mathrm{C} 4$ (log-rank test, $\mathrm{P}=0.644$; Fig. 4B). All the available clinicopathological parameters, in addition to data regarding the genetic status and expression levels of TAZ, AXL and CTGF, were subsequently included in a multivariate Cox regression analysis, which demonstrated that tumor stage [stage II, hazard ratio $(\mathrm{HR})=5.232,95 \%$ confidence interval $(\mathrm{CI})=0.717-38.209, \mathrm{P}=0.103$; stage III, $\mathrm{HR}=6.649,95 \%$ $\mathrm{CI}=0.911-48.541, \mathrm{P}=0.062$; and stage $\mathrm{IV}, \mathrm{HR}=29.934,95 \%$ $\mathrm{CI}=3.894-230.075, \mathrm{P}=0.001]$, expression levels of TAZ, AXL and CTGF (intermediate levels, HR $=1.609,95 \%$, $\mathrm{CI}=0.927-2.792, \mathrm{P}=0.091$; and high levels, $\mathrm{HR}=2.646$, 95\% $\mathrm{CI}=1.513-4.627, \mathrm{P}=0.001)$ and deficient $\mathrm{MMR}$ status $(\mathrm{HR}=0.404,95 \% \mathrm{CI}=0.176-0.930, \mathrm{P}=0.033$; Table II $)$ were independent predictors for relapse-free survival in the patient cohort analyzed in the present study.

According to previous studies, tumor stage appears to be the strongest predictor for poor survival in patients with colon cancer (9), while mutations in the BRAF gene have also been demonstrated to be associated with poor prognosis in colon cancer $(10,11)$. The results of the present study indicated that high expression levels of TAZ, AXL and CTGF were associated with these two prognostic factors. However, the prognostic significance of TAZ-AXL-CTGF was solely detected in patients with wild-type BRAF and KRAS tumors. Overexpression of TAZ, AXL and CTGF was also observed to be associated with poor prognosis in patients with $\mathrm{C} 4$ colon cancer subtype (7), indicating that the increased aggressive behavior of this subtype of colon cancer may be TAZ-AXL-CTGF-dependent. Furthermore, the expression signature of TAZ-AXL-CTGF was able to predict the survival of patients with C6 subtype tumors, who also exhibited poor prognosis. Therefore, TAZ, AXL and CTGF appear to participate in the development of $\mathrm{C} 4$ and $\mathrm{C} 6$, the two most aggressive subtypes of colon cancer.

In addition, the expression of TAZ, AXL and CTGF was associated with deficiency in MMR, which is a favorable prognostic marker $(12,13)$. However, the relevance of this association remains unknown. Nonetheless, the mRNA expression levels of TAZ-AXL-CTGF were observed to be associated with survival in patients with MMR-proficient tumors, but not in those with MMR-deficient tumors, suggesting that the mRNA expression levels of TAZ-AXL-CTGF may regulate certain MMR-associated signaling pathways. Since the majority of patients (441 of 519,85\%) in the dataset analyzed in the present study were MMR-proficient, the results obtained suggest that these patients should be further stratified into favorable or unfavorable prognosis groups in order to improve their personalized treatment plan.

In conclusion, the results of this study indicate that the overexpression of the three-gene signature, TAZ-AXL-CTGF, is associated with more aggressive clinicopathological parameters of colon cancer, suggesting that these three genes may be important in colon cancer progression. However, since the present study was retrospective the results are limited, and thus, future in vitro analysis is required to understand the mechanism of TAZ-AXL-CTGF induced colon cancer progression. Subequently, these future 
studies may result in the identification of potential drug targets for colon cancer.

\section{Acknowledgements}

The present study was supported by the University of Macau Start-Up Research Grant (grant no. SRG2014-00006-FHS), Multi-Year Research Grant (grant no. MYRG201500065-FHS) and the Faculty of Health Sciences Fund.

\section{References}

1. Winder $\mathrm{T}$ and Lenz HJ: Molecular predictive and prognostic markers in colon cancer. Cancer Treat Rev 36: 550-556, 2010.

2. Sridharan M, Hubbard JM and Grothey A: Colorectal cancer: How emerging molecular understanding affects treatment decisions. Oncology (Williston Park) 28: 110-118, 2014.

3. Stein A, Hiemer S and Schmoll HJ: Adjuvant therapy for early colon cancer: Current status. Drugs 71: 2257-2275, 2011.

4. Hong W and Guan KL: The YAP and TAZ transcription co-activators: Key downstream effectors of the mammalian Hippo pathway. Semin Cell Dev Biol 23: 785-793, 2012.

5. Yuen HF, McCrudden CM, Huang YH, Tham JM, Zhang X, Zeng Q, Zhang SD and Hong W: TAZ expression as a prognostic indicator in colorectal cancer. PLoS One 8: e54211, 2013.

6. Wang L, Shi S, Guo Z, Zhang X, Han S, Yang A, Wen W and Zhu Q: Overexpression of YAP and TAZ is an independent predictor of prognosis in colorectal cancer and related to the proliferation and metastasis of colon cancer cells. PLoS One 8: e65539, 2013.
7. Marisa L, de Reyniès A, Duval A, Selves J, Gaub MP, Vescovo L, Etienne-Grimaldi MC, Schiappa R, Guenot D, Ayadi M, et al: Gene expression classification of colon cancer into molecular subtypes: Characterization, validation, and prognostic value. PLoS Med 10: e1001453, 2013.

8. di Pietro M, Sabates Bellver J, Menigatti M, Bannwart F, Schnider A, Russell A, Truninger K, Jiricny J and Marra G: Defective DNA mismatch repair determines a characteristic transcriptional profile in proximal colon cancers Gastroenterology 129: 1047-1059, 2005.

9. Compton CC and Greene FL: The staging of colorectal cancer: 2004 and beyond. CA Cancer J Clin 54: 295-308, 2004.

10. Ogino S, Shima K, Meyerhardt JA, McCleary NJ, Ng K, Hollis D, Saltz LB, Mayer RJ, Schaefer P, Whittom R, et al: Predictive and prognostic roles of BRAF mutation in stage III colon cancer: Results from intergroup trial CALGB 89803. Clin Cancer Res 18: 890-900, 2012.

11. Yokota T, Ura T, Shibata N, Takahari D, Shitara K, Nomura M, Kondo C, Mizota A, Utsunomiya S, Muro K and Yatabe Y: BRAF mutation is a powerful prognostic factor in advanced and recurrent colorectal cancer. Br J Cancer 104: 856-862, 2011.

12. French AJ, Sargent DJ, Burgart LJ, Foster NR, Kabat BF, Goldberg R, Shepherd L, Windschitl HE and Thibodeau SN: Prognostic significance of defective mismatch repair and BRAF V600E in patients with colon cancer. Clin Cancer Res 14: 3408-3415, 2008

13. Sinicrope FA, Foster NR, Thibodeau SN, Marsoni S, Monges G, Labianca R, Kim GP, Yothers G, Allegra C, Moore MJ, et al: DNA mismatch repair status and colon cancer recurrence and survival in clinical trials of 5-fluorouracil-based adjuvant therapy. J Natl Cancer Inst 103: 863-875, 2011. 